\title{
Subventricular zone cell migration: lessons from quantitative two-photon microscopy
}

\author{
Rachel James ${ }^{1}$, Yongsoo Kim ${ }^{2}$, Philip E. Hockberger ${ }^{3}$ and Francis G. Szele ${ }^{1 *}$ \\ Department of Physiology, Anatomy and Genetics, University of Oxford, Oxford, UK \\ 2 Cold Spring Harbor Laboratory, Cold Spring Harbor, New York, NY, USA \\ ${ }^{3}$ Department of Physiology, Feinberg School of Medicine, Northwestern University, Chicago, IL, USA
}

\section{Edited by:}

Silvia De Marchis, University of Turin,

Italy

\section{Reviewed by:}

Angelique Bordey, Yale University

School of Medicine, USA

Dennis Steindler, McKnight Brain

Institute of the University of Florida,

USA

\section{${ }^{*}$ Correspondence:}

Francis G. Szele, Department of

Physiology, Anatomy and Genetics, Le

Gros Clark Building, University of

Oxford, South Parks Road, Oxford OX1

3QS, UK.

e-mail:francis.szele@dpag.ox.ac.uk
Neuroblasts born in the adult subventricular zone (SVZ) migrate long distances in the rostral migratory stream (RMS) to the olfactory bulbs where they integrate into circuitry as functional interneurons. As very little was known about the dynamic parameters of SVZ neuroblast migration, we used two-photon time-lapse microscopy to analyze migration in acute slices. This involved analyzing 3D stacks of images over time and uncovered several novel aspects of SVZ migration: chains remain stable, cells can be immotile for extensive periods, morphology does not necessarily correlate with motility, neuroblasts exhibit local exploratory motility, dorsoventral migration occurs throughout the striatal SVZ, and neuroblasts turn at distinctive angles. We investigated these novel findings in the SVZ and RMS from the population to the single cell level. In this review we also discuss some technical considerations when setting up a two-photon microscope imaging system. Throughout the review we identify several unsolved questions about SVZ neuroblast migration that might be addressed with current or emerging techniques.

Keywords: migration, neuroblast, multiphoton, subependymal zone, subependymal layer

\section{SVZ NEUROBLASTS MIGRATE THROUGH THE ROSTRAL MIGRATORY STREAM TO THE OLFACTORY BULBS}

The subventricular zone (SVZ) lining the lateral ventricles is one of the two largest neurogenic areas within the adult mouse brain. The predominant model of the SVZ delineates three neurogenic celltypes: glial fibrillary acidic protein (GFAP+) stem cells, epidermal growth factor receptor (EGFr+) transit amplifying cells, and doublecortin $(\mathrm{Dcx}+)$ neuroblasts. Slowly dividing stem cells generate more rapidly dividing transit amplifying progenitor cells which in turn give rise to postmitotic migratory neuroblasts (Doetsch et al., 1999). Every day tens of thousands of these immature neurons migrate anteriorly along the rostral migratory stream (RMS) to their final destination in the olfactory bulb (OB; Morshead and van der Kooy, 1992; Luskin, 1993; Lois and Alvarez-Buylla, 1994; Carleton et al., 2003). SVZ neuroblasts move at a distance of 5-8 $\mathrm{mm}$ through the RMS making it the longest migratory pathway in the developing or adult brain. They can take 2-6 days to traverse this length and as will be discussed below it is thus far not possible to follow an individual cell during its entire trajectory. Upon arrival in the $\mathrm{OB}$, these immature neurons migrate into the granular and periglomerular layers and differentiate into a variety of interneurons that integrate into the existing neuronal circuitry (Doetsch and Alvarez-Buylla, 1996; Belluzzi et al., 2003; Carleton et al., 2003).

Throughout development immature neurons tend to migrate in two distinct ways. Radial migration depends upon radial glia acting as a guiding structure. This is typified by excitatory neuron movement from the ventricular zone out to the upper layers of the cerebral cortex. The second type is tangential migration which is in some ways opposite to the classical radial migration of immature neurons. Tangential migration is defined by movement across rather than along radial glia, it covers a longer distance and is not limited to postmitotic neurons (Luskin, 1993; Menezes et al., 1995; Kriegstein and Alvarez-Buylla, 2009). Neuroblast migration from the SVZ to the OB is thought to have features of both of these: tangential migration occurs from the SVZ through the RMS, then there is a switch to migrating radially outward into the OB layers even though this radially oriented migration does not depend on radial glia.

During the tangential migration through the RMS, migrating neuroblasts make cell-cell contacts through neuronophilic interactions and organize themselves into long contiguous arrays of cells (Lois et al., 1996). The combination of individual arrays produces a large scale network of longitudinal "chain migration" that feeds into the RMS and is contiguous with the SVZ. In this homotypic migration cells use each other as a migratory substrate where one cell extends a process that the following cells can use to guide and direct their migration. It seems to follow that if all cells were moving simultaneously as the term chain migration implies, they would have no traction on each other. In fact, as elaborated below a significant portion of neuroblasts in the chain are stationary at any given time (Wichterle et al., 1997; Nam et al., 2007), giving their neighbors substrates for adhesion. Direct cell-cell contact between neuroblasts is crucial for migration, of which the originally studied mediator the polysialylated form of neural cell adhesion molecule is expressed at high levels in the SVZ (PSA-NCAM; Szele et al., 1994; Rousselot et al., 1995; Szele and Chesselet, 1996). PSA residues allow the migration of cells by binding to NCAM and reducing cell adhesion (Sadoul et al., 1983). Removal of PSA-NCAM either by enzymes (Ono et al., 1994) or genetic deletion (Tomasiewicz et al., 1993; Cremer et al., 1994) severely disrupts neuronal migration to the OB (Chazal et al., 2000; Hu, 2000). 
The neuroblasts in the chains migrate rostrally though the SVZ and the RMS to the OB ensheathed by a network of astrocytic processes that form "glial tubes." Ensheathment by the glial tubes does not occur until the third postnatal week before which there is a more homogenous network of glial processes (Peretto et al., 1997, 2005). The exact function of glial tubes is currently unknown as chain migration can occur in the absence of glia (Wichterle et al., 1997). High levels of GFAP and vimentin are expressed by these astrocytes which also secret large amounts of extracellular matrix (ECM) proteins such as tenascin and chondroitin sulfate proteoglycan (Gates et al., 1995; Jankovski and Sotelo, 1996; Thomas et al., 1996). ECM cell surface integrin receptors are present on neuroblasts which interact with laminin to guide migration (Murase and Horwitz, 2002; Emsley and Hagg, 2003), in particular $\beta 1$ integrin promotes the formation of chains (Belvindrah et al., 2007). RMS astrocytes have been proposed to serve both as a physical barrier, preventing dispersion into surrounding parenchyma and as a guidance system for tangential neuroblast migration (Jankovski and Sotelo, 1996; Thomas et al., 1996; Peretto et al., 1997; Kaneko et al., 2010).

The study of SVZ neuroblast migration is important for three major reasons. First it is a convenient system within which to study patterns of cell migration and the molecular mechanisms that regulate them. It eliminates difficult exo utero surgeries, comprises an anatomically defined migratory pathway and shares features of healthy embryonic as well as pathological adult migration (i.e., cancer metastasis). Features of typical embryonic migration that SVZ cells share include lamellipodia, nucleokinesis, and chemotaxis. Cell proliferation during migration occurs in SVZ neuroblasts and during interneuron migration to the cerebral cortex. On the other hand SVZ migration has some unusual features only exhibited by a small number of other migratory events. For example chain migration is unusual but is also seen in neural crest cells. Another reason to study SVZ migration is that SVZ cells may become therapeutically relevant and thus understanding the factors that normally keep them in the RMS in healthy brains or that beckon them toward brain injuries is essential. Finally, migration is a fundamental component of postnatal and adult SVZ neurogenesis. Its proper regulation is as important as cell cycle kinetics, differentiation, and survival in maintaining basal rates of neurogenesis.

\section{TWO-PHOTON TIME-LAPSE MICROSCOPY: DYNAMIC STUDIES OF SVZ MIGRATION}

Many studies have used static histologic sections to infer migration routes and behavior of migrating cells. This is clearly not ideal and more direct analyses are favored since one snapshot of a migrating cell can not reveal its dynamic behavior. Other work has relied on confocal microscopy to generate single optical sections over time. For example, studies on SVZ neuroblast migration from the Goldman lab revealed many interesting aspects of SVZ migration, see history of SVZ imaging below and references (Kakita and Goldman, 1999; Kakita, 2001; Suzuki and Goldman, 2003).

Two-photon microscopy offers an alternative approach to confocal microscopy with several inherent advantages. The technique was first reported by Denk et al. (1990) and has become the preferred method for imaging the dynamic properties of living tissue (Denk and Svoboda, 1997; Zipfel et al., 2003; Svoboda and Yasuda, 2006). The two key advantages of this approach are the small excitation volume (typically on the order of $1 \mu \mathrm{m}^{3}$ ) and the long excitation wavelengths that facilitate deep tissue imaging, reduced photobleaching of the fluorophore, and reduced phototoxicity of cells. In short, two-photon microscopy enables long-term imaging of fluorescently labeled neurons deep within tissues and is ideal for fast moving cells.

One of the major obstacles to performing two-photon microscopy is cost. The main difference in cost between confocal and two-photon microscopy is the laser. Whereas confocal lasers are relatively inexpensive, ranging from $\$ 5$ to $\$ 50 \mathrm{~K}$ for suitable visible lasers, two-photon lasers are more complex and thus more expensive. Two-photon microscopy requires pulsed infrared lasers with a minimum output power of roughly $500 \mathrm{~mW}$ for adequate tissue penetration. Such lasers cost $\$ 100 \mathrm{~K}$ or more especially if you want higher output power (up to $3 \mathrm{~W}$ ).

Another obstacle is that custom built two-photon systems are not as turn-key as commercially available confocal microscopes. In particular, changing objectives is straight-forward using confocal microscopy, whereas it requires some technical expertise to change objectives in two-photon microscopy, i.e., if you want to maintain excitation and collection efficiencies. While not an insurmountable obstacle, this generally requires some knowledge of optics, lasers, and beam alignment. For a more detailed description of our twophoton system, please consult (Nam et al., 2007).

\section{TECHNICAL CONSIDERATIONS WHEN SETTING UP A TWO- PHOTON MICROSCOPIC IMAGING SYSTEM}

1 General microscope setup. There are several commercial suppliers of two-photon microscopes, but they are quite expensive due mostly to development costs. If you have the expertise, then building a custom microscope is not difficult and less costly. Either way, you will need an upright microscope and water-dipping objectives to image tissue slices. The type of microscope is less important and more dependent upon your personal preferences and experience. All commercial suppliers make water-dipping objectives.

2 Objective choice. The most important factor in deciding which water-dipping objective to use is deciding how large a surface area you would like to measure and the level of resolution required. This is determined by the magnification of the lens and the numerical aperture, respectively. We use $20 \times$ and $40 \times$ lenses with high N.A. (0.95 and 0.8 , respectively). The high N.A. maximized our excitation and collection efficiencies. A large diameter back aperture was also preferred as this enabled greater laser beam throughput and greater laser power at the sample which facilitated deeper imaging.

3 Laser choice. You need a tunable, pulsed Ti-Sapphire laser with output ranging from at least $800-900 \mathrm{~nm}$ (most fluorophores are excited within this range). The pulse repetition rate should be at least $75 \mathrm{MHz}$ to maximize the energy per pulse and increase the probability that a fluorophore will absorb two-photons nearly simultaneously (in less than $10^{-15} \mathrm{~s}$ ). Such short pulses also minimize heating of the water in the tissue and surrounding areas that occur at these wavelengths. The latter can become a problem when the laser power at the sample exceeds $50 \mathrm{~mW}$. 
4 Scanners, detector, filters. Since two-photon microscopy is essentially a confocal microscope with a different laser, the need for scanners, detectors, and filters is identical. Since the excitation volume is restricted in two-photon microscopy (due to the low probability of a two-photon absorption event), one does not need to restrict the emission through a pinhole as in confocal microscopy. All of the excitation that reaches the detector can be assumed to arise from the excitation volume. Thus, deeper tissue imaging is possible even though the emitted photons are scattered within the tissue on their way to the detector.

5 Acquisition speed. The speed of acquisition is limited by the amount of fluorophore, its excitation properties (dye cross-section), laser power at the sample, and the efficiency of detection. In our experience, GFP-labeled SVZ cells can be easily visualized using a dwell time of $4 \mu$ s per pixel (the time the laser remains in that location), $800 \mathrm{~nm}$ excitation, 5-10 $\mathrm{mW}$, and internal PMT detectors. At this scanning speed, we routinely collect 40-60 Z-planes every $3 \mathrm{~min}$ for $4 \mathrm{~h}$ and even up to $20 \mathrm{~h}$ with no evidence of photobleaching or phototoxicity. Excitation and collection efficiency could be improved with longer wavelength excitation (900 nm) and use of external detectors.

6 Tissue preparation and plane of sectioning. Acute tissue slices can be a very important tool for examining structural and physiological properties of cells both in imaging and electrophysiological studies. They have several advantages as they are relatively easy to prepare and largely maintain the cytoarchitecture of the tissue. Briefly, the brain is dissected from the skull and chilled in oxygenated artificial cerebrospinal fluid (aCSF). Chilling of the tissue is important as it improves the ease of sectioning and reduces stress to the tissue. Sections of $300 \mu \mathrm{m}$ in thickness are then cut at low speed using a vibratome, followed by incubation in $37^{\circ} \mathrm{C}$ oxygenated aCSF for at least $1 \mathrm{~h}$ then back to room temperature until imaging commences. For multiphoton imaging, and indeed confocal imaging, there are two main planes of section for acute slices that are commonly used: coronal and sagittal. The plane of sectioning for imaging is not always obvious and there are many factors to be considered. Coronal slicing allows limited view of the RMS but may be better for imaging migration out of the SVZ. Cutting tissue coronally results in severing of rostral migration, which may scramble neuroblast migration or cause them to migrate out of the slice. Sagittal slices allow visualization of large portions of the RMS especially if they are angled such that they follow the natural course of the SVZ/RMS. To capture most of the RMS in one slice, we cut the rostral portion of the slice slightly more medially than the caudal. Slicing this way also keeps the $\mathrm{OB}$ attached, theoretically maintaining OB chemoattractants. While we find acute slices one of the best methods to preserve tissue integrity without wide spread apoptosis they are short-lived preparations that we have only used experimentally for up to $24 \mathrm{~h}$. Even when the slice preparation and two-photon microscope are optimal, the experimenter has to continuously monitor the automated image capture for slice drift, computer freezes, etc.

For longer experiments organotypic slice culture may prove a better alternative. However, this method should be approached with some caution if it is to be used to address questions about how certain molecules may influence migration patterns. Bonfanti and colleagues have shown that culturing of SVZ containing coronal sections results in significant disruption of slice cytoarchitecture and cell death over the first several days (Armentano et al., 2011). As a consequence of this loss of $3 \mathrm{D}$ structure, neuroblasts disaggregate at the top and bottom of the slice. Long-term culture is also accompanied by astrocytosis and microgliosis. De Marchis et al. (2001) noted the presence of macrophage-like cells in 3 day old organotypic slices (20\%) that was associated with fragility of the slice. As SVZ/RMS cells can express chemokine and cytokine receptors activation of these inflammatory cells may affect migration (Kokovay et al., 2010).

\section{IMAGE ACQUISITION AND IMAGE ANALYSIS}

The first issue is that the slice must always be immersed in solution to prevent damage to the tissue. Second, while making timelapse movies, the focus may change due to downward drift in the $\mathrm{z}$ dimension that can occur due to the weight of the $\mathrm{C}$ ring used to hold it in place (Figure 1A). Third, drift of the slice may occur in the direction of the perfusion flow. Both drift issues can be prevented or significantly decreased by properly anchoring the slice with a $\mathrm{C}$ ring (Figure 1A) and by leaving the slice in the perfusion chamber for $1 \mathrm{~h}$ before acquiring movies so that the slice has time to adhere to the top of the platform. Most $\mathrm{z}$ drift occurs during this $1 \mathrm{~h}$ time period which also allows acclimatization of the slice to the aCSF and the temperature. We routinely look for drift during the preincubation period by rapidly using a $5 \times$ objective lens to visualize GFP+ cells

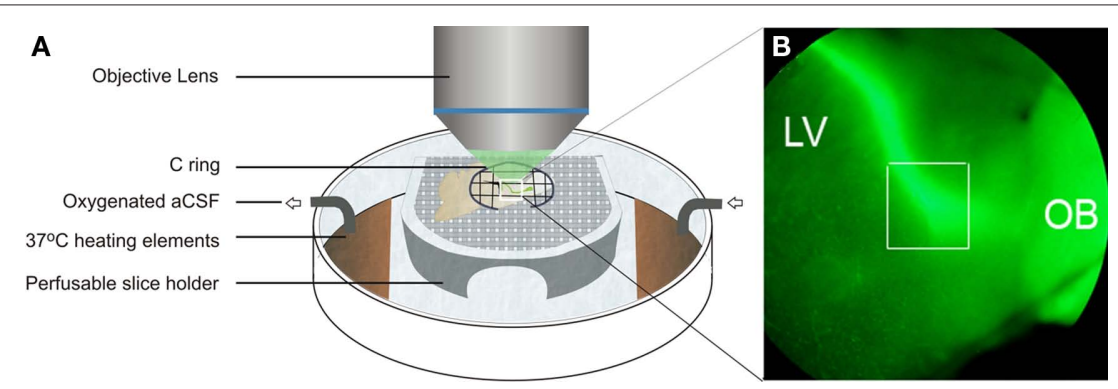

FIGURE 1 | Depiction of a GFP+ slice in the imaging chamber. (A) The slice rests on a perfusable platform and is held in place by the weight of a $\mathrm{C}$ ring. (B) Low magnification image of a nestin-GFP slice showing labeled cells migrating from the SVZ above the lateral ventrical (LV) through the RMS (boxed area) into the olfactory bulb (OB). Adapted from Kim et al. (2009). 
(Figure 1B). Temperature control is an important parameter since fluctuations in the temperature can cause expansion and contraction of the tissue and thus unnecessary loss of focus. Several stage and objective warmers are available, but we have primarily used a Bioptechs chamber (Figure 1A). This chamber utilizes current resistance in a thin metallic film across the bottom to generate heat that is easily regulated. Oxygenated aCSF is then rapidly $(1 \mathrm{ml} /$ min) perfused over and under the slice which is held in place with a simple $\mathrm{C}$ ring fashioned from tungsten wire and cross-hatched with nylon fibers. Once the slice is in place we search for optimal fields with a $5 \times$ lens and then switch to $40 \times$ and confirm that sufficient signal to noise ratios can be attained.

We have used Olympus Confocal software (Fluoview) to capture images (Figure 2A). Fluoview is rather easy to use and allows control over the number of optical slices, the distance between slices, the digital zoom and initial Kallman filtering. Once the timelapse is completed files are imported into Volocity (Improvision) software and are rendered into 3D images (Figure 2B). Although Volocity can run into $\$ 10-30 \mathrm{~K}$ depending on the suite of modules one chooses, we have found it ideal for subsequent filtering (Figure 2C) and especially for tracking and quantifying cells in 3D. Although the program requires a high-performance computer with large memory capacity, the computer should still only amount to $10-20 \%$ of cost of the image analysis system. We have found it extremely useful to routinely convert movies from Volocity into Quicktime files for archiving, rapid examination of parameters and for presentations (Figure 2D). To image the tracks taken by migrating cells we frequently export data to Graphis (Kylebank Software) which is convenient for visualizing the pathways from multiple angles (Figure 2E).

\section{A BRIEF HISTORY OF SVZ NEUROBLAST TIME-LAPSE IMAGING}

Studies by Joseph Altman in the 1960s using tritiated thymidine clearly delineated separate proliferative and migratory cells in the SVZ and RMS (Altman, 1962, 1969). It was not until the 1990s that live imaging of SVZ neuroblast migration was achieved. Kakita and Goldman labeled early postnatal rat SVZ cells with GFP encoding retroviruses and used confocal time-lapse microscopy to image their migration in the SVZ and into adjacent nuclei (Kakita and Goldman, 1999; Kakita, 2001). The majority of these cells were glial progenitors and interestingly they took many dramatic turns, similar to those we have subsequently observed in mouse neuroblasts (Martinez-Molina et al., 2010). The turns could result from de novo growth of processes or from bending of pre-existing processes (Kakita, 2001). Furthermore, the authors noted significant dorsoventral movements within the wall of the lateral ventricle, a behavior we have also documented and which suggests that significant intermixing of SVZ lineages could take place. Subsequent studies from the Goldman lab used similar techniques and directly visualized rostral migration of SVZ neuroblasts to the OB, contrasting that route with migration of SVZ glioblasts to the corpus callosum, cerebral cortex, and striatum (Suzuki and Goldman, 2003). Remarkably many neuroblasts could reverse their direction by $180^{\circ}$, even though the large majority eventually migrate from the SVZ to the OB. In that study, the authors also noted obvious boundaries to migration of neuroblasts into the adjacent structures that glial precursors readily moved into (Suzuki and Goldman, 2003). To this day it is unclear what the molecular basis of such different behaviors may be.

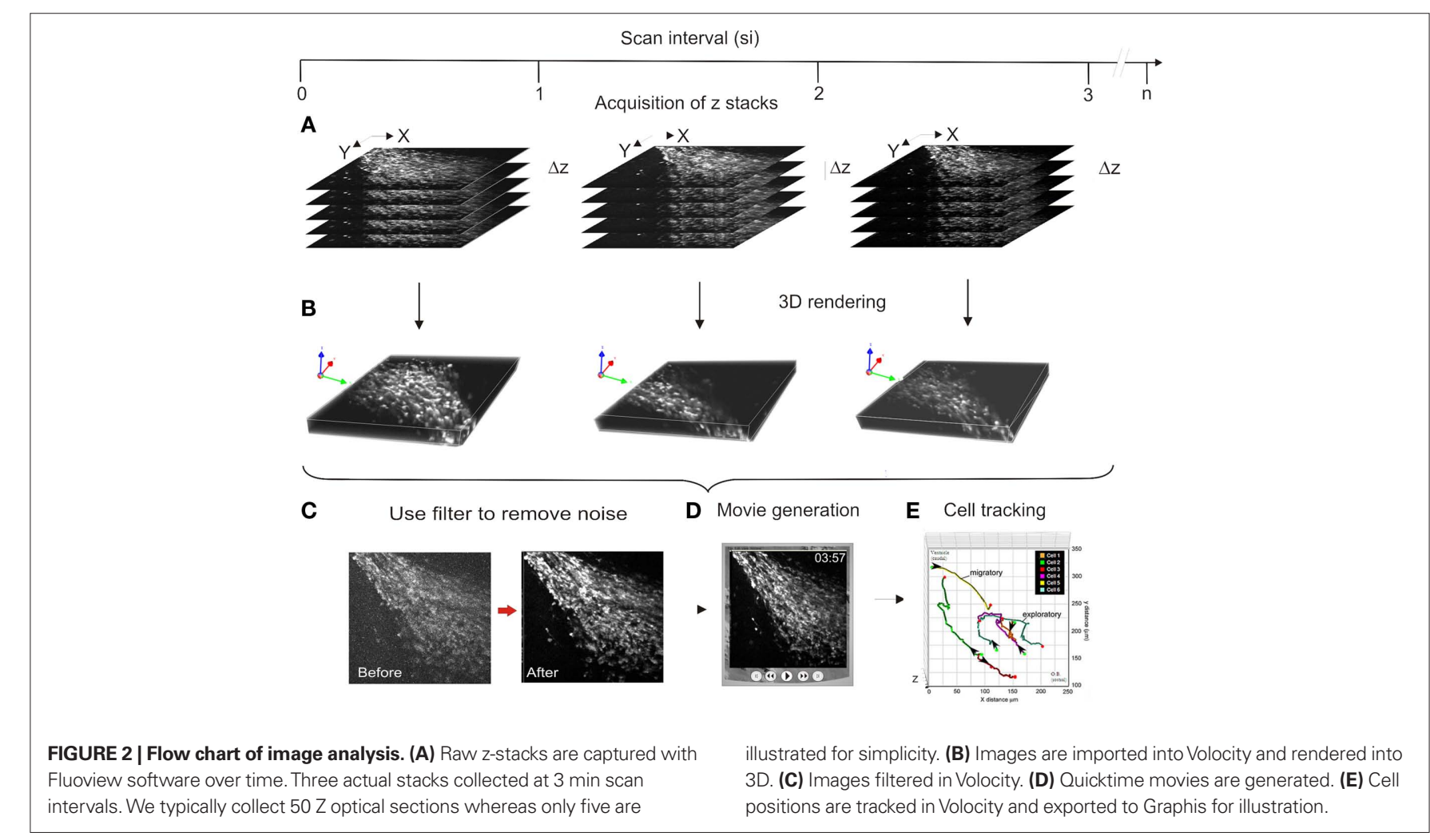


Later dynamic imaging studies of neuroblasts by Puche and colleagues involved injecting a fluorescent dye called cell tracker green (CTG) into the lateral ventricles (De Marchis et al., 2001,2004a). This dye is able to diffuse though the wall of the ventricle and label migratory cells within the SVZ, irrespective of their location in the SVZ. As the CTG does not passively spread into the RMS any cells labeled cells found in the RMS after several days can only have migrated there from the SVZ (Kim et al., 2009). CTG is enzymatically modified upon entry is rendered impermeable to the plasma membrane and thus becomes trapped in the cell. CTG fills the cytoplasm of cells allowing visualization of morphology such as leading and trailing processes and their dynamic changes during migration. Using this method, De Marchis et al. (2001) showed it is possible to track SVZ neuroblasts along the RMS to the OB using confocal microscopy. They also identified a second ventrocaudal migratory stream originating at the elbow between the vertical and horizontal limbs of the RMS that is present during the first postnatal week (De Marchis et al., 2004a). While the CTG technique has several obvious strengths it also has some inherent problems. In order to study specific SVZ populations cell subtypes need to be identified after dynamic imaging with post hoc immunohistochemistry as the dye does not label specific cells. Another important consideration is that CTG is not water soluble and requires dimethyl sulfoxide (DMSO) as a carrier. Although few signs of toxicity have been reported (De Marchis et al., 2001), it cannot be ruled out that DMSO induces changes within the SVZ molecular niche. In our laboratory limiting the concentration of CTG and the volume of DMSO injected were both essential to avoid periventricular degeneration (Kim et al., 2009).

One of the central difficulties in studying the SVZ is distinguishing the multiple cell types found in it and in the RMS (Figure 3A). Recently several lines of transgenic mice have become available in which fluorescent proteins are expressed under the control of celltype specific promoters (Figure 3B), for an excellent review see Lacar et al. (2010). These mouse lines have opened up the ability to ask specific questions about migration and motility of distinct SVZ/ RMS cell populations. Two main lines are available for neuroblasts: Dcx-eGFP mice and Gad65-eGFP mice (Figure 3B; Nam et al., 2007). Dcx is expressed by SVZ neuroblasts and is a microtubule-associated protein necessary for their migration (Koizumi et al., 2006; Ocbina et al., 2006). The Dcx-GFP mouse by Gensat (Gong et al., 2003) clearly shows chains and for the first time allowed visualization of migration within the entire array of cells (Nam et al., 2007). However, since SVZ cell density is very high it is difficult to discern individual cells. Gad65, the 65-kDa isoform of glutamic acid decarboxylase (Gad) is expressed by a subset of neuroblasts as they migrate through the SVZ and RMS (De Marchis et al., 2004b; Hamilton et al., 2008). In Gad65-eGFP mice this subset of Dcx+ and PSA-NCAM+ neuroblasts probably represents the more mature cells. By labeling individual cells within arrays this facilitates morphological and quantitative analyses.

Several nestin-eGFP lines have been generated that label similar precursor cell populations (Figure 3B; Yamaguchi et al., 2000; Kawaguchi et al., 2001; Mignone et al., 2004; Walker et al., 2011). Nestin is an intermediate filament protein widely used to identify neural stem and progenitor cells (Lendahl et al., 1990; Thomas et al., 1996; Doetsch et al., 1997). These nestin-GFP lines label various subsets of all SVZ and RMS cells. We have used a particularly bright nestin-eGFP mouse that clearly labels individual neuroblasts (Figure 3B; Nam et al., 2007; Walker et al., 2011). This can be advantageous insofar as individual fluorescent cells can be clearly visualized making it easy to track them in $3 \mathrm{D}$, frame by frame. Another very useful line is from the Enikolopov laboratory at Cold Spring Harbor, we have it termed "CSH-Nestin-GFP" (Mignone et al., 2004). In these mice, GFAP+ stem-like cells and transit amplifying cells in the SVZ are labeled with bright GFP and neuroblasts with dim GFP (Figure 3B) allowing them to be distinguished in the same slice (Kim et al., 2009).
A

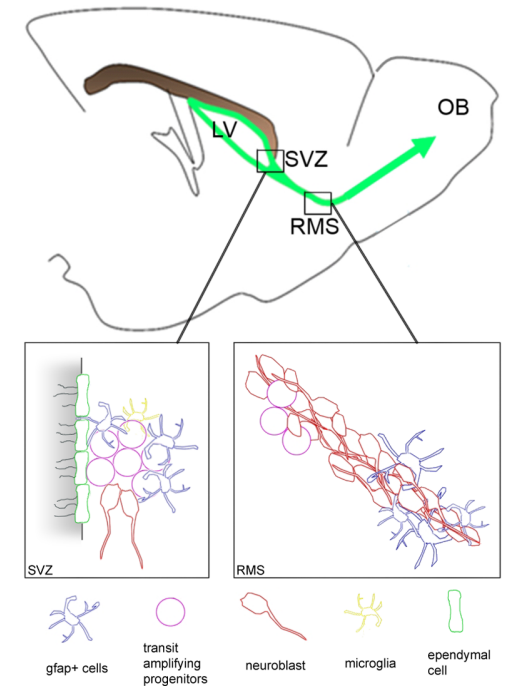

FIGURE 3 | Schematic of the SVZ, RMS, and cell subtypes labeled in different transgenic lines. (A) Low magnification view of a sagittal section showing location of the SVZ, RMS, and OB. SVZ cell subpopulations are depicted by different shapes and colors. Boxed areas show cytoarchitectural

\section{B}
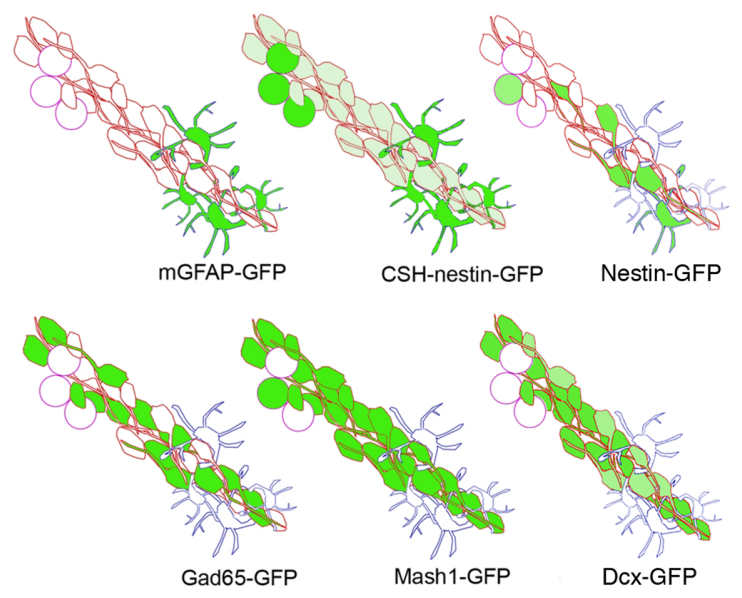

organization of the SVZ (left, coronal plane) and RMS (right, sagittal plane). (B) Examples of mouse lines used to label SVZ subpopulations. Green indicates SVZ cell subtypes labeled as well as the relative intensity of GFP fluorescence. (Modified from Nam et al., 2007 and Kim et al., 2009). 


\section{VISUALIZING CHAIN MIGRATION}

The SVZ/RMS pathway contains a diverse and unique molecular environment with the presence of soluble, ECM and adhesion molecules (Thomas et al., 1996; Conover et al., 2000; Hedin-Pereira et al., 2000). Currently we have little understanding of how these molecules coordinate to regulate chemoattraction, chemorepulsion, motogenesis, speed, detachment from chains and cell adhesion. The RMS has no clear anatomical guidance structure so how do these cells orientate and navigate through this complex environment? One of the main goals of using two-photon imaging is to understand the fundamental rules and factors involved in governing these subcomponents of cell motility.

The first studies using dynamic imaging to demonstrate homotypic or chain migration cultured SVZ explants and produced time-lapse movies of the outgrowth of chains (Wichterle et al., 1997). Interestingly, this study demonstrated that chains formed and neuroblasts migrated without the ensheathing glial tubes seen in the RMS, suggesting astrocytic tubes may not be necessary for chain formation and RMS migration. Neuroblasts leap-frogged over each other while a certain percentage were stationary, similar to what our 2-P time-lapse studies subsequently showed in slices (Nam et al., 2007). Although cell culture and tissue explants are important tools for studying the mechanisms of cell migration and differentiation, these models involve significant alterations to the normal anatomical and functional relationships of the cellular elements in the SVZ, RMS, and OB. Conclusions drawn from these may not represent the in vivo situation.

By using time-lapse two-photon imaging it is possible to study these chains at the population level and image these arrays for up to $24 \mathrm{~h}$. Using the Dcx-GFP mouse which labels all neuroblasts (Figure 3B), movies can be taken that allow the arrays to be visualized in their entirety. Imaging reveals that the arrays have the appearance of cells streaming along clearly delineated, well defined pathways in which the direction of migration closely matches the orientation of the array. In vitro studies suggest that these chains are not stable but change shape by dissipating and reforming (Wichterle et al., 1997). However, our ex vivo studies suggest that this does not seem to be the case as the chains remain very stable over hours of imaging and cells very rarely err from the SVZ/RMS pathway into the surrounding tissue. This may be due to contactmediated inhibition with neurons and oligodendrocytes in the striatum and corpus callosum that induce lamellipodial collapse.

The large numbers of cells born in the SVZ migrate rostrally through the RMS to the OB. As expected, imaging shows that neuroblast arrays are oriented rostrocaudally immediately under the corpus callosum, in the dorsal SVZ, and the majority of rostral migration probably occurs in this subregion (Nam et al., 2007). It is currently unknown what sets up this rostral direction of migration but it has been suggested it may be diffusible chemoattractants secreted by the OB (Liu and Rao, 2003; Ng et al., 2005) and chemorepellents from the caudal septum (Hu and Rutishauser, 1996) working in combination. Other studies however have shown that these long distance cues may not be required as rostral migration continues in the absence of the OB (Jankovski and Sotelo, 1996; Kirschenbaum et al., 1999). We have also observed that removal of the OB before imaging does not change the migration or motility of neuroblasts within the RMS (Szele Laboratory, unpublished observations).
Ependymal ciliary beating has been proposed to set up the flow of CSF around the ventricles and thereby establish caudal to rostral chemotactic gradients of factors secreted by the choroid plexus that can direct SVZ migration (Sawamoto et al., 2006). Loss of this flow in Tg737orpk mice with defective cilia results in neuroblast chain disorientation and a loss of their longitudinal arrangement. In the slice preparations used for two-photon imaging this seems unlikely to explain migration guidance as constant perfusion of aCSF would dissipate any gradient. Also, the direction of aCSF flow from experiment to experiment had no obvious effect on migration direction. It is more likely that gradients of factors such as Slit2 (Sawamoto et al., 2006) are important during developmental stages when morphogenic gradients have to be established across shorter distances, but are not vital for rostral migration throughout adulthood.

While migration in the dorsal SVZ occurs largely in the rostral direction, evidence suggests that the orientation of migration may be different in other regions of the SVZ. Previous studies have shown that in the ventral portions of the SVZ Dcx+ arrays are orientated dorsoventrally (Yang et al., 2004; Nam et al., 2007) and ventral migration may occur into the anterior striatal SVZ (Sawamoto et al., 2006). Using two-photon time-lapse to visualize dorsoventral migration, we showed that it occurs throughout the entire striatal SVZ and not only in the anterior subregion (Nam et al., 2007). The ventral SVZ is characterized by radial glia like cells whose processes fan out into the ventral forebrain regions (Sundholm-Peters et al., 2004). In neonatal, and to a lesser extent adult mice, Dcx+ cells may use these radial processes to emigrate dorsoventrally to the ventral forebrain and produce neurons in the olfactory tubercles, islands of Calleja and nucleus accumbens (De Marchis et al., 2004a; Sundholm-Peters et al., 2004; Yang et al., 2004). Our finding of this widespread dorsoventral migration raises several interesting questions regarding differences between dorsal and ventral SVZ cells. Do dorsal and ventral cells intermix or are they two distinct populations? Are there differences in the molecular mechanisms that create the varying array orientation and motility directions? Future imaging studies may reveal different migration mechanisms in SVZ subregions.

\section{MORPHOLOGY AND MOTILITY OF SVZ NEUROBLASTS}

A large amount of what is known about the SVZ has been inferred from histological studies including several ideas about neuroblast migration. Many studies performing static studies have identified migrating cells by using a common "migratory morphology" represented by unipolar or bipolar cells that display a leading process and trailing edge (De Marchis et al., 2001). Leading process orientation is often used as a sole indicator of directionality of cell migration (Szele and Cepko, 1996; Sawamoto et al., 2006). It has long been assumed that there is a strict relationship between this morphology and motility without direct evidence to prove this is the case. A major impetus for conducting time-lapse imaging studies was to directly study this relationship between morphology and motility.

Time-lapse confocal studies examining the migration of CTG labeled cells (De Marchis et al., 2001; Bovetti et al., 2007a) demonstrate that many migratory neurons exhibit a long leading process with a short trailing process. As described above, these studies have difficulties in resolving the processes as intensity and exposure time have to be kept short to prevent phototoxicity. The use of 
confocal for studying migration is limited as images can only be taken on average every $10-15$ min over a limited period of time (De Marchis et al., 2001; Bovetti et al., 2007a) and pathways of migration between these imaging frames may not be observed. Furthermore, by examining CTG+ cells that have migrated from the SVZ into the RMS imaging is biased toward cells that have migrated long distances and may not accurately represent local migration.

We took $\mathrm{Z}$-stacks every 3 min with two-photon time-lapse providing much greater temporal resolution than confocal time-lapse. 3 min intervals were necessary to raster in the $X Y$ dimension across $512 \times 512$ pixels $\left(0.125 \mathrm{~mm}^{2}\right)$ in 50 optical sections ( $Z$ dimension; Nam et al., 2007). However in preparations that label large number of cells smaller volumes and shorter time intervals between 3D frames should be very possible. Using two-photon imaging of nestin-eGFP mice we unexpectedly found that many cells that had typical migratory morphology were in fact stationary in the RMS (19\%), with an even larger percentage stationary in the SVZ (40\%) (Nam et al., 2007; Kim et al., 2009). Imaging over several hours showed that these stationary cells could have stable cell bodies but dynamic processes that changed in length, orientation, or disappeared completely. Alternatively, the processes remained stable but the cell bodies exhibit subtle morphological changes. It is possible that these cells represent SVZ GFAP+ astrocytes and the dynamic processes interacted directly with the motile cells. While many cells with "migratory morphology" were indeed motile more surprising was the presence of motile cells that had no processes or were multipolar. This was particularly important as close to half of the cells in the RMS were motile (Nam et al., 2007) and would have been classed as stationary using histological methods.

\section{SPEED OF SVZ NEUROBLAST MIGRATION}

A fundamental feature of migration is the average speed at which cells travel, as speed must be well regulated throughout the RMS to synchronize cell production in the SVZ with integration in the OB. Any changes in this speed can have dramatic effects, as is shown when PSA is removed from NCAM reducing migration speeds so cells accumulate in the RMS and the size of the OB diminishes (Chazal et al., 2000). The earliest studies on neuroblast migratory speed used retroviral lineage tracers in rats and calculated speed by measuring the distance between a cell's point of origin and its final destination after a certain time (Luskin and Boone, 1994). These studies calculated average speed to be around $20-30 \mu \mathrm{m} / \mathrm{h}$. These are inherently inaccurate measurements, as they make the assumption that cells move in a straight line and at constant velocity. Measurements of neuroblast migration from SVZ explants show a much higher speed of migration, around $122 \mu \mathrm{m} / \mathrm{h}$ (Wichterle et al., 1997). This is most probably due to the lack of complex cytoarchitecture that cells have to migrate through in situ. Also, there are no glial tubes in the in vitro system, suggesting that astrocytes may slow migration speed.

Migrating cells within the RMS use 3D and often very complex pathways which cannot be fully appreciated using standard confocal methodology. Analysis of confocal and early two-photon movies (Koizumi et al., 2006) was achieved by collapsing the z-stack to produce a $2 \mathrm{D}$ image, meaning that the path taken by the cell appears much shorter and may have lead to underestimations of speeds. By using two-photon imaging, cell migration can be tracked in $3 \mathrm{D}$ by recording the $X, Y$, and $Z$ coordinates over time as described above. Using this method we were able to show that by examining complex 3D movements, speed can be calculated with more accuracy (Nam et al., 2007; Kim et al., 2009). Generally average speeds measured using two-photon were faster than those reported using confocal due to the larger distance measured as traveled. Interestingly, Gad65-GFP cells migrated at a faster speed $(71 \mu \mathrm{m} / \mathrm{h})$ than nestin-eGFP+ cells $(52 \mu \mathrm{m} / \mathrm{h})$. Gad65+ neuroblasts are thought to be relatively differentiated in comparison to nestin+ cells (De Marchis et al., 2001). This raised an intriguing possibility that older neuroblasts may be distinguished from younger ones by using speed as a phenotypic characterization.

Studies using confocal imaging of organotypic slices allowed the first dynamic imaging of neuroblast migration in situ in the RMS and show that cells migrate in a saltatory manner representative of nucleokinesis (Kakita and Goldman, 1999; Suzuki and Goldman, 2003). This movement is characterized by elongation of the leading process followed by translocation of the nucleus (Nadarajah et al., 2003; Tsai and Gleeson, 2005) resulting in alternating periods of higher and lower migration rates when based on cell body movement. There is large variation in measured average migration speeds for different confocal studies (De Marchis et al., 2001; Bovetti et al., 2007b; Platel et al., 2008). Some of this may be due to different ages of the animals with some focusing on neonates and other using adult animals. Alternatively speeds may be affected by temperature, a correlation that we have not examined since we always work at physiological temperatures. Many changes occur in the SVZ during the first postnatal week with retraction of radial glial processes as they differentiate into SVZ astrocytes and ependymal cells (Merkle et al., 2004). Bovetti et al. (2007b) report that cells migrate quicker at P21 than at P5 and that younger mice lack defined glial tubes (Peretto et al., 1997, 2005) and chains and migrate more as individual cells.

\section{SVZ NEUROBLASTS DISPLAY COMPLEX MOTILITY PATTERNS}

The detailed information that can be gathered using two-photon methods means that fundamental but previously undetected motility behaviors can be detected and studied. One approach to this is to use a "migratory index" which is a simple indication of motility complexity that is independent of speed ( $\mathrm{MI}=$ net distance/total distance). By grouping cells based on migratory index we found the RMS is comprised of at least three distinct subpopulations of cells: migratory, exploratory, and intermediate (Nam et al., 2007). The migratory cells have fairly direct, straight migration pathways with little deviation. In contrast exploratory cells move in a local exploratory fashion.

It is possible that exploratory behavior may be an epiphenomenon within the ex vivo environment caused by the disruption of molecular gradients. We think this is unlikely, and that exploratory motility is not due to unhealthy slices or unusual mouse strains because it is consistently observed in all slices and in different transgenic lines. In addition, multipolar migration in the embryonic cortex (Tabata and Nakajima, 2003) closely resembles exploratory behavior. However that work was also performed in cultured slices, which may cause loss of local diffusion factors and thereby induce these behaviors. If exploratory motility does also occur in vivo what is its function? One possibility is it serves as a prelude to injury induced emigration from the SVZ/RMS into surrounding tissues (Goings et al., 2004; Sundholm-Peters et al., 2005; Dizon 
et al., 2006) as our observations suggest that exploratory behavior increases after cortical injury (Szele laboratory, unpublished observations). Exploratory behavior may provide a means of sampling the environment to receive molecular cues. Further studies are required to see if migratory and exploratory cells express different cell surface signaling molecules, allowing intrinsic regulation of motility in a given microenvironment. It is important to point out that neuroblasts could switch behaviors from exploratory to migratory. Although we have yet to perform a thorough analysis of these switches thus far it seems that they are random.

Using two-photon time-lapse our laboratory has also found that EGFr signaling may be another mechanism controlling neuroblast motility. Neuroblasts that expressed low levels of EGFr tended to move in exploratory patterns. Neuroblasts show an inverse correlation between EGFr and Dcx/PSA-NCAM/bIII-tubulin expression, suggesting that EGFr is gradually lost as neuroblasts mature. Epidermal growth factor (EGF) regulates cell division in the SVZ in vivo (Reynolds and Weiss, 1992; Kuhn et al., 1997; Gritti et al., 1999) and is important for neurosphere generation in vitro. In the presence of transforming growth factor alpha (TGFa), an EGFrselective agonist the percentage of motile cells decreased by approximately 40\% (Kim et al., 2009). A very important consequence of this finding is with regards to the use of organotypic slices to measure aspects of motility as EGF is sometimes included as a constituent of culture media (Zhang et al., 2009).

\section{SVZ NEUROBLASTS EXHIBIT STEREOTYPIC BEHAVIORS}

Previous reports in rats had documented cells migrating countercurrent to each other within the chains, with some cells migrating caudally (Kakita and Goldman, 1999; Suzuki and Goldman, 2003). In both Gad65-GFP and nestin-GFP mice around 25\% of cells turned and migrated caudally. Interestingly there was no difference in average speeds of cells that migrated caudally compared to cells that migrated only rostrally (Nam et al., 2007). These changes in direction were accompanied by complex and dynamic movements of the leading process and lamellipodium. Kakita and Goldman (1999) described how glia progenitors changed direction by branching and curving of the leading process or growth of a new process at the opposite pole of the cell body. Using two-photon time-lapse we show that neuroblasts could change direction by either polarity reversal or sequential bending of the leading process as previously described or by a newly observed method of branching of the leading process (Martinez-Molina et al., 2010).

During forward migration cells also exhibited complex methods of turning even in the absence of direction changes. Most cells deviated from straight paths through bending of the leading process close to the cell body ( $47 \%$ of turns), we termed this P-bending. We also observed bending of the distal leading process (30\% of turns) or branching of the leading process ( $23 \%$ of turns). Remarkably, the angle of bending $\left(128^{\circ}\right)$ and of branching $\left(101^{\circ}\right)$ was very consistent from cell to cell, which may imply that similar molecular mechanisms control these movements regardless of the direction of movement or type of turn (Martinez-Molina et al., 2010).

The SVZ is a mosaic of cells of different lineages which raises the possibility they exhibit different patterns of motility (Merkle et al., 2007; Young et al., 2007). Interestingly, individual neuroblasts do not seem to be restricted to a specific type of turning behavior. Indeed, the same cell can not only switch between exploratory and migratory behaviors but can also change direction in multiple patterns. This suggests that certain behaviors are not specific to different lineages. As behaviors are not cell specific does one type of behavior predict or preclude another? What remains to be answered are the specific sequences and molecular mechanisms that govern neuroblast turning.

Given that neuroblast chains are largely established through neuronophilic interactions neural progenitor cells may influence each other's motility to maintain chain migration. Within the interior of chains, neuroblasts form contacts with each other, while on the exterior, cells encounter astrocytes forming the glial tube in addition to other neuroblasts. It is currently unknown whether in vivo cells are able to switch chain position and shift between the interior and exterior. Further analysis of cell migration patterns may reveal whether individual turning behaviors are associated with the cell's position in the interior versus exterior of the chain. For example, neuroblasts that stop movement in the interior of the chains may be carried along with the other cells, while on the exterior neuroblasts may be able to form stable contacts with glial tubes that anchor them in a stationary position. Contact with astrocytes may then induce turning or direction reversal through contact inhibition as seen in other systems. In vitro experiments have shown that SVZ neuroblasts repel astrocytes via Slit/Robo signaling in dissociated cells (Kaneko et al., 2010). However, in SVZ explants which lack ensheathing glial tubes a cell moving in a chain may become stationary, and by changing the orientation of its leading process begin to move in the opposite direction (Wichterle et al., 1997). Furthermore, the addition of striatal, septal, or OB explants alongside SVZ explants does not influence the direction of migration (Jankovski et al., 1998). Together this would suggest that extrinsic directional cues may not be required for cell turning patterns but that turning is regulated by mechanisms that are intrinsic to the neuronal progenitors.

\section{NEUROBLAST DIVISION AND TRANSIT AMPLIFYING PROGENITOR MIGRATION}

An essential feature of SVZ biology which has been largely neglected in most dynamic migration studies is the interaction between proliferation and cell motility. During tangential migration through the RMS, cells continue to divide and initiate neuronal maturation (Menezes et al., 1995; Coskun et al., 2007). Retroviral studies demonstrate that cells dividing in the RMS have a much longer cell cycle time than those dividing in the SVZ (Smith and Luskin, 1998). It will be interesting to study whether daughter cells pause during or after division or whether daughter cells migrate immediately. It is also unknown if sibling cells share the same migratory behaviors.

Recent evidence suggested that some SVZ progenitor cells may be motile (Aguirre et al., 2004, 2005). If multipotent stem cells or rapidly dividing progenitor cells have migratory potential they may be able to migrate to injury sites. Since they are not as faterestricted as neuroblasts, they may be more reparative. We found motile nestin-GFP+ cells that did not express Dcx, suggesting stem cells or progenitor SVZ cells could be motile (Nam et al., 2007) since Dcx is thought to be expressed only in SVZ neuroblasts. In addition, cells with SVZ progenitor cell characteristics emigrated to the striatum in a model of injury supplemented with growth factor infusion (de Chevigny et al., 2008). 
To directly address whether SVZ stem or progenitor cells could be migratory we performed two-photon imaging using the $\mathrm{CSH}$ nestin-GFP mouse. To further distinguish between the stem and progenitor cells two-photon movies were made with the mGFAPGFP mouse which labels a subset of SVZ astrocytes (Figure 3B; Kuzmanovic et al., 2003; Kim et al., 2009). After examination of over 800 CSH-Nestin-GFP bright and 65 mGFAP-GFP+ cells none moved suggesting that GFAP+ astrocyte-like stem cells are normally static within the SVZ (Kim et al., 2009).

To examine the progenitor population we imaged the NestinGFP mouse and immediately after imaging the tissue was fixed and followed by post hoc double immunohisotchemistry for Dcx and Mash1. Mash1 is a transcription factor characteristic of SVZ transit amplifying progenitors which can be easily co-localized with GFP post hoc. The confocal image of the immunohistochemistry was matched with the last frame of the two-photon movie and Mash1+/GFP+ cells that were identified as being Dcx-negative identified. By following the movies backward the motility of these cells could be tracked. We never saw any motile Mash1+ cells implying that Mash1+ progenitor cells do not migrate from the SVZ into the RMS. Currently there are no transgenic lines that are specific

\section{REFERENCES}

Aguirre, A., Rizvi, T. A., Ratner, N., and Gallo, V. (2005). Overexpression of the epidermal growth factor receptor confers migratory properties to nonmigratory postnatal neural progenitors. J. Neurosci. 25, 11092-11106.

Aguirre, A.A., Chittajallu, R., Belachew, S., and Gallo, V. (2004). NG2-expressing cells in the subventricular zone are type C-like cells and contribute to interneuron generation in the postnatal hippocampus. J. Cell Biol. 165, 575-589.

Altman, J. (1962). Are new neurons formed in the brains of adult mammals? Science 135, 1127-1128.

Altman, J. (1969). Autoradiographic and histological studies of postnatal neurogenesis. IV. Cell proliferation and migration in the anterior forebrain, with special reference to persisting neurogenesis in the olfactory bulb. $J$. Comp. Neurol. 137, 433-457.

Armentano, M., Canalia, N., Crociara, P., and Bonfanti, L. (2011). Culturing conditions remarkably affect viability and organization of mouse subventricular zone in ex vivo cultured forebrain slices. J. Neurosci. Methods (In Press).

Barretto, R. P., Messerschmidt, B., and Schnitzer, M. J. (2009). In vivo fluorescence imaging with high-resolution microlenses. Nat. Methods6,511-512.

Belluzzi, O., Benedusi, M., Ackman, J., and LoTurco, J. J. (2003). Electrophysiological differentiation of new neurons in the olfactory bulb. J. Neurosci. 23, 10411-10418.

Belvindrah, R., Hankel, S., Walker, J., Patton, B. L., and Muller, U. (2007).
Betal integrins control the formation of cell chains in the adult rostral migratory stream. J. Neurosci. 27, 2704-2717.

Bovetti, S., Bovolin, P., Perroteau, I., and Puche, A. C. (2007a). Subventricular zone-derived neuroblast migration to the olfactory bulb is modulated by matrix remodelling. Eur. J. Neurosci. 25, 2021-2033.

Bovetti, S., Hsieh, Y. C., Bovolin, P., Perroteau, I., Kazunori, T., and Puche, A. C. (2007b). Blood vessels form a scaffold for neuroblast migration in the adult olfactory bulb. J. Neurosci. 27, 5976-5980.

Carleton, A., Petreanu, L. T., Lansford, R., Alvarez-Buylla, A., and Lledo, P. M. (2003). Becoming a new neuron in the adult olfactory bulb. Nat. Neurosci. 6, 507-518.

Chazal, G., Durbec, P., Jankovski, A., Rougon, G., and Cremer, H. (2000). Consequences of neural cell adhesion molecule deficiency on cell migration in the rostral migratory stream of the mouse. J. Neurosci. 20, 1446-1457.

Conover,J.C., Doetsch, F., Garcia-Verdugo, J. M., Gale, N. W., Yancopoulos, G. D., and Alvarez-Buylla, A. (2000). Disruption of Eph/ephrin signaling affects migration and proliferation in the adult subventricular zone. Nat. Neurosci. 3, 1091-1097.

Coskun, V., Falls, D. L., Lane, R., Czirok, A., and Luskin, M. B. (2007). Subventricular zone neuronal progenitors undergo multiple divisions and retract their processes prior to each cytokinesis. Eur. J. Neurosci. 26, 593-604.

to the transit amplifying progenitor cells. The Rockefeller Gensat Project have produced a Mash1-GFP mouse but immunostaining showed that the GFP expression pattern did not mirror that of the endogenous Mash 1 protein (Figure 3B), but more represented that of Dcx labeling of the neuroblast population (Kim et al., 2009).

In summary we have learned much about cellular patterns of SVZ neuroblast migration and the mechanisms that regulate them. We contend that use of two-photon time-lapse microscopy to study this fascinating migratory system is still in its early days. Several emerging strategies may further harness the deep potential of two-photon microscopy. These include time-lapse of whole mount preparations to study migration at the population level and intravital microscopy using a variety of lenses (Flusberg et al., 2008; Barretto et al., 2009). The latter will be important to confirm current findings from slice preparations in vivo.

\section{ACKNOWLEDGMENTS}

We would like to thank members of the Szele lab and M. Majumdar for reading and suggestions on the manuscript. Francis G. Szele supported by NIH RO1 NS-42253. Rachel James supported by a Wellcome Trust studentship.

Cremer, H., Lange, R., Christoph, A., Plomann, M., Vopper, G., Roes, J., Brown, R., Baldwin, S., Kraemer, P. Scheff, S., Barthels, D., Rajewsky, K., and Wille, W. (1994). Inactivation of the N-CAM gene in mice results in size reduction of the olfactory bulb and deficits in spatial learning. Nature 367, 455-459.

de Chevigny, A., Cooper, O., Vinuela, A., Reske-Nielsen, C., Lagace, D. C., Eisch, A. J., and Isacson, O. (2008). Fate mapping and lineage analyses demonstrate the production of a large number of striatal neuroblasts after transforming growth factor alpha and noggin striatal infusions into the dopaminedepleted striatum. Stem Cells 26, 2349-2360.

De Marchis, S., Fasolo, A., and Puche, A. C. (2004a). Subventricular zone-derived neuronal progenitors migrate into the subcortical forebrain of postnatal mice. J. Comp. Neurol. 476, 290-300.

De Marchis, S., Temoney, S., Erdelyi, F. Bovetti, S., Bovolin, P., Szabo, G., and Puche, A. C. (2004b). GABAergic phenotypic differentiation of a subpopulation of subventricular derived migrating progenitors. Eur. J. Neurosci. 20, 1307-1317.

De Marchis, S., Fasolo, A., Shipley, M. and Puche, A. (2001). Unique neuronal tracers show migration and differentiation of SVZ progenitors in organotypic slices. J. Neurobiol. 49, 326-338.

Denk, W., Strickler, J. H., and Webb, W. W. (1990). Two-photon laser scanning fluorescence microscopy. Science 248, 73-76.
Denk, W., and Svoboda, K. (1997). Photon upmanship: why multiphoton imaging is more than a gimmick. Neuron 18, 351-357.

Dizon, M. L., Shin, L., Sundholm-Peters, N. L., Kang, E., and Szele, F. G. (2006). Subventricular zone cells remain stable in vitro after brain injury. Neuroscience 142, 717-725.

Doetsch, F., and Alvarez-Buylla,A. (1996). Network of tangential pathways for neuronal migration in adult mammalian brain. Proc. Natl. Acad. Sci. U.S.A. 93, 14895-14900.

Doetsch, F., Garcia-Verdugo, J. M., and Alvarez-Buylla, A. (1997). Cellular composition and three-dimensional organization of the subventricular germinal zone in the adult mammalian brain. J. Neurosci. 17, 5046-5061.

Doetsch, F., Garcia-Verdugo, J. M., and Alvarez-Buylla, A. (1999). Regeneration of a germinal layer in the adult mammalian brain. Proc. Natl. Acad. Sci. U.S.A. 96, 11619-11624.

Emsley, J. G., and Hagg, T. (2003). Alpha6betal integrin directs migration of neuronal precursors in adult mouse forebrain. Exp. Neurol. 183, 273-285.

Flusberg, B. A., Nimmerjahn, A., Cocker, E. D., Mukamel, E. A., Barretto, R. P., Ko, T. H., Burns, L. D., Jung, J. C., and Schnitzer, M. J. (2008). High-speed, miniaturized fluorescence microscopy in freely moving mice. Nat. Methods 5, 935-938.

Gates, M. A., Thomas, L. B., Howard, E. M., Laywell, E. D., Sajin, B., Faissner, A. Gotz, B., Silver, J., and Steindler, D. A (1995). Cell and molecular analysis of 
the developing and adult mouse subventricular zone of the cerebral hemispheres. J. Comp. Neurol. 361, 249-266.

Goings, G. E., Sahni, V., and Szele, F. G. (2004). Migration patterns of subventricular zone cells in adult mice change after cerebral cortex injury. Brain Res. 996, 213-226.

Gong, S., Zheng, C., Doughty, M. L., Losos, K., Didkovsky, N., Schambra, U. B., Nowak, N. J., Joyner, A., Leblanc, G., Hatten, M. E., and Heintz, N. (2003). A gene expression atlas of the central nervous system based on bacterial artificial chromosomes. Nature 425 , 917-925.

Gritti, A., Frolichsthal-Schoeller, P., Galli, R., Parati, E. A., Cova, L., Pagano, S. F., Bjornson, C. R., and Vescovi, A. L. (1999). Epidermal and fibroblast growth factors behave as mitogenic regulators for a single multipotent stem cell-like population from the subventricular region of the adult mouse forebrain. J. Neurosci. 19, 3287-3297.

Hamilton, K. A., Parrish-Aungst, S., Margolis, F. L., Erdelyi, F., Szabo, G., and Puche,A.C. (2008). Sensory deafferentation transsynaptically alters neuronal GluR1 expression in the external plexiform layer of the adult mouse main olfactory bulb. Chem. Senses 33, 201-210.

Hedin-Pereira, C., deMoraes, E. C., Santiago, M. F., Mendez-Otero, R., and Lent, R. (2000). Migrating neurons cross a reelin-rich territory to form an organized tissue out of embryonic cortical slices. Eur. J. Neurosci. 12, 4536-4540.

$\mathrm{Hu}, \mathrm{H}$. (2000). Polysialic acid regulates chain formation by migrating olfactory interneuron precursors. J. Neurosci. Res. 61, 480-492.

Hu, H., and Rutishauser, U. (1996). A septum-derived chemorepulsive factor for migrating olfactory interneuron precursors. Neuron 16, 933-940.

Jankovski, A., Garcia, C., Soriano, E., and Sotelo, C. (1998). Proliferation, migration and differentiation of neuronal progenitor cells in the adult mouse subventricular zone surgically separated from its olfactory bulb. Eur. J. Neurosci. 10, 3853-3868.

Jankovski, A., and Sotelo, C. (1996). Subventricular zone-olfactory bulb migratory pathway in the adult mouse: cellular composition and specificity as determined by heterochronic and heterotopic transplantation. J. Comp. Neurol. 371, 376-396.

Kakita, A. (2001). Migration pathways and behavior of glial progenitors in the postnatal forebrain. Hum. Cell 14, 59-75.

Kakita, A., and Goldman, J. E. (1999). Patterns and dynamics of SVZ cell migration in the postnatal forebrain: monitoring living progenitors in slice preparations. Neuron 23, 461-472.

Kaneko, N., Marin, O., Koike, M., Hirota, Y., Uchiyama, Y., Wu, J. Y., Lu, Q., Tessier-Lavigne, M., Alvarez-Buylla, A., Okano, H., Rubenstein, J. L., and Sawamoto, K. (2010). New neurons clear the path of astrocytic processes for their rapid migration in the adult brain. Neuron 67, 213-223.

Kawaguchi, A., Miyata, T., Sawamoto, K., Takashita, N., Murayama, A., Akamatsu, W., Ogawa, M., Okabe, M., Tano, Y., Goldman, S. A., and Okano, H. (2001). Nestin-EGFP transgenic mice: visualization of the self-renewal and multipotency of CNS stem cells. Mol. Cell. Neurosci. 17, 259-273.

Kim, Y., Comte, I., Szabo, G., Hockberger, P., and Szele, F. G. (2009). Adult mouse subventricular zone stem and progenitor cells are sessile and epidermal growth factor receptor negatively regulates neuroblast migration. PLoS ONE 4, e8122. doi: 10.1371/journal. pone. 0008122

Kirschenbaum, B., Doetsch, F., Lois, C., and Alvarez-Buylla, A. (1999). Adult subventricular zone neuronal precursors continue to proliferate and migrate in the absence of the olfactory bulb. J. Neurosci. 19, 2171-2180.

Koizumi, H., Higginbotham, H., Poon, T., Tanaka, T., Brinkman, B. C., and Gleeson, J. G. (2006). Doublecortin maintains bipolar shape and nuclear translocation during migration in the adult forebrain. Nat. Neurosci. 9 , 779-786.

Kokovay, E., Goderie, S., Wang, Y., Lotz, S., Lin, G., Sun, Y., Roysam, B., Shen, Q., and Temple, S. (2010). Adult SVZ Lineage cells home to and leave the vascular niche via differential responses to SDF1/CXCR4 signaling. Cell Stem Cell 7, 163-173.

Kriegstein, A., and Alvarez-Buylla, A. (2009). The glial nature of embryonic and adult neural stem cells. Annu. Rev. Neurosci. 32, 149-184.

Kuhn, H. G., Winkler, J., Kempermann, G., Thal, L. J., and Gage, F. H. (1997). Epidermal growth factor and fibroblast growth factor-2 have different effects on neural progenitors in the adult rat brain. J. Neurosci. 17, 5820-5829.

Kuzmanovic, M., Dudley, V. J., and Sarthy, V. P. (2003). GFAP promoter drives Muller cell-specific expression in transgenic mice. Invest. Ophthalmol. Vis. Sci. 44, 3606-3613.

Lacar, B., Young, S. Z., Platel, J. C., and Bordey, A. (2010). Imaging and recording subventricular zone progenitor cells in live tissue of postnatal mice. Front. Neurosci. 4:43. doi: 10.3389/fnins.2010.00043
Lendahl, U., Zimmerman, L. B., and McKay, R. D. (1990). CNS stem cells express a new class of intermediate filament protein. Cell 60, 585-595.

Liu, G., and Rao, Y. (2003). Neuronal migration from the forebrain to the olfactory bulb requires a new attractant persistent in the olfactory bulb. $J$. Neurosci. 23, 6651-6659.

Lois, C., and Alvarez-Buylla, A. (1994). Long-distance neuronal migration in the adult mammalian brain. Science 264, 1145-1148.

Lois, C., Garcia-Verdugo, J. M., and Alvarez-Buylla, A. (1996). Chain migration of neuronal precursors. Science 271, 978-981.

Luskin, M. B. (1993). Restricted proliferation and migration of postnatally generated neurons derived from the forebrain subventricular zone. Neuron 11, 173-189.

Luskin, M. B., and Boone, M. S. (1994). Rate and pattern of migration of lineally-related olfactory bulb interneurons generated postnatally in the subventricular zone of the rat. Chem. Senses 19, 695-714.

Martinez-Molina, N., Kim, Y., Hockberger, P., and Szele, F. G. (2010). Rostral migratory stream neuroblasts turn and change directions in stereotypic patterns. Cell Adh. Migr. 5, 83-95.

Menezes, J. R., Smith, C. M., Nelson, K. C., and Luskin, M. B. (1995). The division of neuronal progenitor cells during migration in the neonatal mammalian forebrain. Mol. Cell. Neurosci. 6, 496-508.

Merkle, F. T., Mirzadeh, Z., and AlvarezBuylla,A. (2007). Mosaic organization of neural stem cells in the adult brain. Science 317, 381-384.

Merkle, F. T., Tramontin, A. D., GarciaVerdugo, J. M., and Alvarez-Buylla, A. (2004). Radial glia give rise to adult neural stem cells in the subventricular zone. Proc. Natl. Acad. Sci. U.S.A. 101, 17528-17532.

Mignone, J. L., Kukekov, V., Chiang, A. S., Steindler, D., and Enikolopov, G. (2004). Neural stem and progenitor cells in nestin-GFP transgenic mice. J. Comp. Neurol. 469, 311-324.

Morshead, C. M., and van der Kooy, D. (1992). Postmitotic death is the fate of constitutively proliferating cells in the subependymal layer of the adult mouse brain. J. Neurosci. 12, 249-256

Murase, S., and Horwitz, A. F. (2002). Deleted in colorectal carcinoma and differentially expressed integrins mediate the directional migration of neural precursors in the rostral migratory stream. J. Neurosci. 22, 3568-3579.

Nadarajah, B., Alifragis, P., Wong, R. O. L., and Parnavelas, J. G. (2003). Neuronal migration in the develop- ing cerebral cortex: observations based on real-time imaging. Cereb. Cortex 13 , 607-611.

Nam, S. C., Kim, Y., Dryanovski, D., Walker, A., Goings, G., Woolfrey, K., Kang, S.S., Chu, C., Chenn, A., Erdelyi, F., Szabo, G., Hockberger, P., and Szele, F. G. (2007). Dynamic features of postnatal subventricular zone cell motility: a two-photon time-lapse study. J. Comp. Neurol. 505, 190-208.

Ng, K. L., Li, J. D., Cheng, M. Y., Leslie, F. M., Lee, A. G., and Zhou, Q. Y. (2005). Dependence of olfactory bulb neurogenesis on prokineticin 2 signaling. Science 308, 1923-1927.

Ocbina, P. J., Dizon, M. L., Shin, L., and Szele, F. G. (2006). Doublecortin is necessary for the migration of adult subventricular zone cells from neurospheres. Mol. Cell. Neurosci. 33, 126-135.

Ono, K., Tomasiewicz, H., Magnuson, T., and Rutishauser, U. (1994). N-CAM mutation inhibits tangential neuronal migration and is phenocopied by enzymatic removal of polysialic acid. Neuron 13, 595-609.

Peretto, P., Giachino, C., Aimar, P., Fasolo, A., and Bonfanti, L. (2005). Chain formation and glial tube assembly in the shift from neonatal to adult subventricular zone of the rodent forebrain. J. Comp. Neurol. 487, 407-427.

Peretto, P., Merighi, A., Fasolo, A., and Bonfanti, L. (1997). Glial tubes in the rostral migratory stream of the adult rat. Brain Res. Bull. 42, 9-21.

Platel, J. C., Heintz, T., Young, S., Gordon, V., and Bordey, A. (2008). Tonic activation of GLUK5 kainate receptors decreases neuroblast migration in whole-mounts of the subventricular zone. J. Physiol. 586, 3783-3793.

Reynolds, B. A., and Weiss, S. (1992). Generation of neurons and astrocytes from isolated cells of the adult mammalian central nervous system. Science 255, 1707-1710.

Rousselot, P., Lois, C., and Alvarez-Buylla, A. (1995). Embryonic (PSA) N-CAM reveals chains of migrating neuroblasts between the lateral ventricle and the olfactory bulb of adult mice. $J$. Comp. Neurol. 351, 51-61.

Sadoul, R., Hirn, M., Deagostini-Bazin, H., Rougon, G., and Goridis, C. (1983). Adult and embryonic mouse neural cell adhesion molecules have different binding properties. Nature 304, 347-349.

Sawamoto, K., Wichterle, H., GonzalezPerez, O., Cholfin, J. A., Yamada, M. Spassky, N., Murcia, N. S., GarciaVerdugo, J. M., Marin, O., Rubenstein, J. L., Tessier-Lavigne, M., Okano, H., and Alvarez-Buylla, A. (2006). New neurons follow the flow of cerebrospinal fluid in the adult brain. Science 311, 629-632. 
Smith, C. M., and Luskin, M. B. (1998). Cell cycle length of olfactory bulb neuronal progenitors in the rostral migratory stream. Dev. Dyn. 213, 220-227.

Sundholm-Peters, N. L., Yang, H. K., Goings, G. E., Walker, A. S., and Szele, F. G. (2004). Radial glia-like cells at the base of the lateral ventricles in adult mice. J. Neurocytol. 33, 153-164.

Sundholm-Peters, N. L., Yang, H. K., Goings, G. E., Walker, A. S., and Szele, F. G. (2005). Subventricular zone neuroblasts emigrate toward cortical lesions. J. Neuropathol. Exp. Neurol.64, 1089-1100.

Suzuki, S. O., and Goldman, J. E. (2003). Multiple cell populations in the early postnatal subventricular zone take distinct migratory pathways: a dynamic study of glial and neuronal progenitor migration. J. Neurosci. 23, 4240-4250.

Svoboda, K., and Yasuda, R. (2006). Principles of two-photon excitation microscopy and its applications to neuroscience. Neuron 50, 823-839.

Szele, F. G., and Cepko, C. L. (1996). A subset of clones in the chick telencephalon arranged in rostrocaudal arrays. Curr. Biol. 6, 1685-1690.

Szele, F. G., and Chesselet, M. F. (1996). Cortical lesions induce an increase in cell number and PSA-N-CAM expres- sion in the subventricular zone of adults rats. J. Comp. Neurol. 368, 439-454.

Szele, F. G., Dowling, J. J., Gonzales, C., Theveniau, M., Rougon, G., and Chesselet, M. F. (1994). Pattern of expression of highly polysialylated neural cell adhesion molecule in the developing and adult rat striatum. Neuroscience 60, 133-144.

Tabata, H., and Nakajima, K. (2003). Multipolar migration: the third mode of radial neuronal migration in the developing cerebral cortex. J. Neurosci. 23, 9996-10001.

Thomas, L. B., Gates, M. A., and Steindler, D. A. (1996). Young neurons from the adult subependymal zone proliferate and migrate along an astrocyte, extracellular matrix-rich pathway. Glia 17 , $1-14$.

Tomasiewicz, H., Ono, K., Yee, D., Thompson, C., Goridis, C., Rutishauser, U., and Magnuson, T. (1993). Genetic deletion of a neural cell adhesion molecule variant (N-CAM-180) produces distinct defects in the central nervous system. Neuron 11, 1163-1174.

Tsai, L. H., and Gleeson, J. G. (2005). Nucleokinesis in neuronal migration. Neuron 46, 383-388.

Walker, A., Goings, G., Kim, Y., Miller, R. J., Chen, A., Szele, F. (2011). Nestin reporter transgene labels multiple central nervous system precursor cells. Neural Plast. 2010, Article ID 894374.

Wichterle, H., Garcia-Verdugo, J. M., and Alvarez-Buylla, A. (1997). Direct evidence for homotypic, glia-independent neuronal migration. Neuron 18 779-791.

Yamaguchi, M., Saito, H., Suzuki, M., and Mori, K. (2000). Visualization of neurogenesis in the central nervous system using nestin promoter-GFP transgenic mice. Neuroreport 11, 1991-1996.

Yang, H. K., Sundholm-Peters, N. L., Goings, G. E., Walker, A. S., Hyland, K and Szele, F. G. (2004). Distribution of doublecortin expressing cells near the lateral ventricles in the adult mouse brain. J. Neurosci. Res. 76, 282-295.

Young, K. M., Fogarty, M., Kessaris, N., and Richardson, W. D. (2007). Subventricular zone stem cells are heterogeneous with respect to their embryonic origins and neurogenic fates in the adult olfactory bulb. $J$. Neurosci. 27, 8286-8296.

Zhang, R. L., Chopp, M., Gregg, S. R., Toh, Y., Roberts, C., Letourneau, Y., Buller, B., Jia, L., Davarani, S. P.N., and Zhang, Z. G. (2009). Patterns and dynamics of subventricular zone neuroblast migration in the ischemic striatum of the adult mouse. J. Cereb. Blood Flow Metab. 29, 1240-1250.

Zipfel, W. R., Williams, R. M., and Webb, W.W. (2003). Nonlinear magic: multiphoton microscopy in the biosciences. Nat. Biotechnol. 21, 1369-1377.

Conflict of Interest Statement: The authors declare that the research was conducted in the absence of any commercial or financial relationships that could be construed as a potential conflict of interest.

Received: 26 January 2011; accepted: 24 February 2011; published online: 21 March 2011.

Citation: James R, Kim Y, Hockberger PE and Szele FG (2011) Subventricular zone cell migration: lessons from quantitative two-photon microscopy. Front. Neurosci. 5:30. doi: 10.3389/fnins.2011.00030

This article was submitted to Frontiers in Neurogenesis, a specialty of Frontiers in Neuroscience.

Copyright (c) 2011 James, Kim, Hockberger and Szele. This is an open-access article subject to an exclusive license agreement between the authors and Frontiers Media SA, which permits unrestricted use, distribution, and reproduction in any medium, provided the original authors and source are credited. 